\title{
Reducción de color con cloruro de magnesio en soluciones con colorantes comerciales
}

\author{
Mercedes Lucero Chávez*, Mario Esparza Soto*, María del Carmen Jiménez Moleón*, \\ Daury García Pulido* y Sahara Gabriela Rojas Reyes*
}

Recepción: 25 de febrero de 2016
Aceptación: 26 de abril de 2017

*Universidad Autónoma del Estado de México, México. Correos electrónicos: mluceroc@uaemex.mx; mesparzas@uaemex.mx; mcjimenezm@uaemex.mx; dgarciap@uaemex.mx; sahara_gabriela@hotmail.com Se agradece a la Universidad Autónoma del Estado de México por el apoyo prestado para el desarrollo de este estudio y a los comentarios de los árbitros de la revista.
Resumen. Se experimentó con diferentes concentraciones de cloruro de magnesio

$\left(\mathrm{MgCl}_{2}\right)$ como coagulante para reducir el color en soluciones con colorantes comerciales: negro, azul y café. Los parámetros analizados fueron $\mathrm{pH}$, color, demanda química de oxígeno (DQO) y sólidos suspendidos totales (SST) de acuerdo con las Normas Mexicanas. Como prueba complementaria se agregó carbón activado a las muestras para reducir el color residual después de la coagulación-floculación. La reducción de los valores de color y DQO fue mayor cuando se adicionó más cantidad de $\mathrm{MgCl}_{2}(1.8 \mathrm{~g} / \mathrm{L})$. Los porcentajes de reducción fueron $\geq 90 \%$ para el color $y>50 \%$ para la DQO. Los SST aumentaron con la adición de $\mathrm{MgCl}_{2}$. El carbón activado eliminó el color residual.

Palabras clave: reducción, colorantes comerciales, cloruro de magnesio.

\begin{abstract}
Reduction of Color with Magnesium Chloride in Commercial Dye Solutions

Abstract. The aim of this study was to experiment with different concentrations of magnesium chloride $\left(\mathrm{MgCl}_{2}\right)$ as a coagulant to reduce the color in commercial dye solutions: black, blue and brown. The parameters analyzed according to the Mexican Standards were $\mathrm{pH}$, color, chemical oxygen demand (COD) and total suspended solids (TSS). Additionally, an activated carbon was added to the samples to reduce residual color after the coagulation-flocculation test. The reduction of color and COD values was greater when more amount of $\mathrm{MgCl}_{2}(1.8 \mathrm{~g} / \mathrm{L})$ was added. The reduction percentages were $\geq 90 \%$ in the color and $>50 \%$ for COD. The Tss increased with the addition of $\mathrm{MgCl}_{2}$. Activated carbon removed residual color.
\end{abstract}

Keywords: reduction, commercial dyes, magnesium chloride.

\section{Introducción}

En el tratamiento de agua potable el proceso de coagulaciónfloculación es primordial, mientras que en el de agua residual es opcional. En el área de tratamiento de agua potable la clarificación del agua con agentes coagulantes ha sido practicada desde tiempos antiguos, en la cual se usaba una variedad de sustancias, en especial las semillas trituradas.

La coagulación tiene como objetivo la desestabilización de los coloides en el agua, por lo que neutraliza las cargas que se mantienen en el vital líquido. Esto se logra por lo general añadiendo coagulantes químicos y aplicando agitación constante. Los coagulantes más usados son sulfato de aluminio, sulfato ferroso y cloruro férrico.

Por otro lado, dentro de las aplicaciones en las que se destaca el empleo del proceso de la coagulación-floculación se encuentran las siguientes:

a) La remoción de turbiedad orgánica o inorgánica que no puede sedimentar rápidamente.

b) La remoción de color verdadero y aparente.

c) La eliminación de bacterias, virus y organismos patógenos susceptibles de ser separados por coagulación.

d) La destrucción de algas y plancton en general.

e) La eliminación de sustancias productoras de sabor. 
El proceso de coagulación-floculación se lleva a cabo en las plantas de tratamiento de aguas residuales para mejorar la eficiencia de los procesos primarios y secundarios, donde se usan coagulantes con diferentes grados de eficiencia. Los costos de los coagulantes son altos y se asume que de estos depende más de $80 \%$ de la tasa de remoción de sólidos. Los procesos mecánicos como la tasa de corte contribuyen de manera significativa a la eficiencia de remoción de sólidos; sin embargo, la tasa de corte no está implementada en las plantas de tratamiento. En este estudio se realizaron pruebas de coagulación-floculación con colorantes comerciales y dos dosis de cloruro de magnesio $\left(\mathrm{MgCl}_{2}\right)$ a $\mathrm{pH} 12$ con el objetivo de obtener la reducción del color de las soluciones preparadas con colorantes comerciales.

La estructura química de los colorantes utilizados se desconoce; a pesar de esto, ha de considerarse porque influye en la intensidad del color. Para que una sustancia proporcione color debe tener por lo menos dos grupos presentes en su molécula: el grupo cromóforo (compuesto cromógeno) y el grupo auxocromo (Macy, 2005).

El grupo cromóforo está constituido por algunos grupos de átomos que presentan uno o más enlaces insaturados. $\mathrm{Su}$ presencia es la responsable de generar color. Ejemplos de cromóforos fuertes: quinoide $\left(=\mathrm{C}_{6} \mathrm{H}_{4}=\right)$, azo $(-\mathrm{N}=\mathrm{N}-)$, nitroso $(-\mathrm{N}=\mathrm{O})$ y cromóforos débiles: doble enlace carbono-

Cuadro 1.
\begin{tabular}{|lccc} 
Condiciones de trabajo para las pruebas de coagulación-floculación \\
\hline Muestra & $\begin{array}{c}\mathrm{NaOH} \\
(\mathrm{g} / \mathrm{L})\end{array}$ & $\begin{array}{c}\mathrm{MgCl}_{2} \\
(\mathrm{~g} / \mathrm{L})\end{array}$ & $\begin{array}{c}\text { Carbón activado } \\
\text { (g CAP/40 mL muestra) }\end{array}$ \\
\hline Negro elegante & 0 & 0 & 0.4020 \\
Negro 1 & 0.4845 & 0.4493 & 0.1014 \\
Negro 2 & 0.4245 & 1.8224 & 0.1032 \\
Azul profundo & 0 & 0 & 0.401 \\
Azul 1 & 0.5426 & 0.4627 & 0.1014 \\
Azul 2 & 0.5026 & 1.8168 & 0.1032 \\
Café tabaco & 0 & 0 & 0.4013 \\
Café 1 & 0.4990 & 0.4840 & 0.1003 \\
Café 2 & 0.5140 & 1.8009 & 0.1006 \\
\hline Nota: CAP = carbón activado en polvo. & & \\
Fuente: elaboración propia. & & \\
\hline
\end{tabular}

Tabla 1. Parámetros, técnicas analíticas y equipos utilizados.

\begin{tabular}{|lll} 
Parámetro & \multicolumn{1}{c}{ Técnica analítica } & \multicolumn{1}{c}{ Equipo } \\
\hline pH & $\begin{array}{l}\text { NMX-AA-008-SCFI-2011 } \\
\text { (DOF, 2011) }\end{array}$ & $\begin{array}{l}\text { Medidor de pH, marca Thermoscientific, modelo Orion } \\
\text { Star A211 }\end{array}$ \\
Color & Método 8025, Hach & $\begin{array}{l}\text { Espectofotómetro marca Hach, modelo DR 5000 } \\
\text { DQO }\end{array}$ \\
& $\begin{array}{l}\text { NMX-AA-030/2-SCFI-2011 } \\
\text { (DOF, 2013) }\end{array}$ & $\begin{array}{l}\text { Digestor marca Hach, modelo DR B200 } \\
\text { Espectofotómetro marca Hach, modelo DR 5000 }\end{array}$ \\
SST & NMX-AA-034-SCFI-2001 & Estufa marca Lab-line, modelo Imperial V \\
& (DOF, 2016) & Mufla marca Barnestead, modelo Thermolyne 6000 \\
\hline Fuente: elaboración propia. & \\
\hline
\end{tabular}

carbono $(>\mathrm{C}=\mathrm{C}<)$, compuesto nitro $\left(-\mathrm{NO}_{2}\right)$, aldehídos, cetonas, ésteres $(>\mathrm{C}=\mathrm{O})$ (Macy, 2005).

El grupo auxocromo es un grupo de átomos o radicales libres con carga positiva que tienen la propiedad de intensificar la acción de un grupo cromóforo dentro de la misma molécula de una sustancia. Ejemplos de auxocromos: grupos ácidos: acetilo $(-\mathrm{COOH})$, hidroxilo $(-\mathrm{OH})$, sulfonilo $\left(-\mathrm{SO}_{3} \mathrm{H}\right)$. Grupos básicos: amino $\left(-\mathrm{NH}_{2}\right)$ y amino mono sustituido (-NHR) (Macy, 2005).

Tomando en cuenta lo anterior, se indican en el anexo A1 las características de algunos colorantes similares a los utilizados en este trabajo.

\section{Metodología}

Las pruebas de coagulación-floculación se realizaron con soluciones de colorantes comerciales: negro elegante $(3.019 \mathrm{~g} / 3$ L), azul profundo $(3.001 \mathrm{~g} / 3 \mathrm{~L})$ y café tabaco $(3.010 \mathrm{~g} / 3 \mathrm{~L})$.

La prueba de coagulación-floculación se llevó acabo tomando como base a Bao-Yu et al. (2007). Antes de la prueba se adicionó hidróxido de sodio en masa (aproximadamente $0.4 \mathrm{~g} \mathrm{NaOH} / \mathrm{L}$ ) para ajustar el $\mathrm{pH}$ cercano a 12.0.

El cloruro de magnesio que se utilizó como coagulante en las pruebas de coagulación-floculación fue grado reactivo. Se adicionó aproximadamente 0.4 y $1.8 \mathrm{~g}$ por litro.

Las condiciones de trabajo en las pruebas de coagulaciónfloculación fueron las siguientes: mezcla rápida $150 \mathrm{rpm}$ por 1 min, mezcla lenta $45 \mathrm{rpm}$ por 20 min y la sedimentación de $30 \mathrm{~min}$ antes de muestrear el sobrenadante.

Bao-Yu el al. (2007) indican 10 min de sedimentación, posteriormente tomar una alícuota del sobrenadante y centrifugar por 5 min para obtener un líquido claro. En este caso sólo se dejó sedimentar por 30 min y se realizaron las pruebas debido a que en campo no sería factible centrifugar. Después de la sedimentación el color persistió, por lo que se efectuó una prueba complementaria con carbón activado. Después de la prueba de coagulación-floculación, a las muestras se les adicionó carbón activado, se agitaron manualmente y se filtraron con papel Whatman 41. Las condiciones de trabajo se muestran el cuadro 1.

Los parámetros analizados para las diferentes pruebas de coagulaciónfloculación fueron $\mathrm{pH}$, color, demanda química de oxígeno (DQO) y sólidos suspendidos totales (SST) de acuerdo con las Normas Mexicanas y método Hach (tabla 1). Se utilizó un equipo de prueba de jarras marca Temsa, modelo JTR-1010. 
Al finalizar las pruebas de coagulación-floculación se elaboró un análisis espectral de la muestra antes y después del proceso de coagulación-floculación.

\section{Resultados y discusión}

El uso de los reactivos de $\mathrm{NaOH}$ y $\mathrm{MgCl}_{2}$ en las pruebas de coagulación-floculación da lugar a que se forme el $\mathrm{Mg}(\mathrm{OH})_{2}$, que provee una estructura adsortiva para la reducción de color (Bao-Yu et al., 2007). La cantidad de $\mathrm{NaOH}$ que se adicionó fue suficiente para elevar el $\mathrm{pH}$ cercano a 12.0 para el tratamiento. La dosis cercana de $0.4 \mathrm{~g}$ de $\mathrm{MgCl}_{2}$ se seleccionó porque de esta dosis Bao-Yu et al. (2007) obtuvieron reducción de color por arriba de $80 \%$. Pero teniendo en cuenta que ellos centrifugaron la muestra después de la prueba de coagulación-floculación, se decidió adicionar 4.5 veces más de $\mathrm{MgCl}_{2}$ al no centrifugar en esta experimentación.

Con el pH de 12.0 Bao-Yu et al. (2007) obtuvieron la más alta reducción de color (90-97\%). Ellos llevaron a cabo el estudio con agua residual sintética con colorante $(1 \mathrm{~g} / 10 \mathrm{~L}$ de agua destilada). Hai Tan et al. (2000) concluyeron que a pH entre 10.5 y 11 , y con una dosis de $3 \mathrm{~g} \mathrm{MgCl}_{2} / \mathrm{L}$, fueron capaces de reducir el color más de $90 \%$ en una solución de azul brillante EBRA $(1 \mathrm{~g} / \mathrm{L})$. En este estudio el color se redujo entre 90 y $97 \%$, porcentaje calculado de acuerdo con Somasiri et al. (2008). A pesar del alto porcentaje de reducción de color, el mismo persistió.

La adición de dos cantidades de $\mathrm{MgCl}_{2}(0.4$ y $1.8 \mathrm{~g} / \mathrm{L})$ y una de $\mathrm{NaOH}(0.4 \mathrm{~g} / \mathrm{L})$ dio lugar a la interrogante de cuál sería la cantidad óptima de reactivos a utilizar en las pruebas de coagulación-floculación. Se hizo un análisis de estequiometría para conocer cuál fue el reactivo limitante y cuál fue el reactivo en exceso, y de esta manera, en trabajos posteriores, adicionar el $\mathrm{NaOH}$ y el $\mathrm{MgCl}_{2}$ en cantidades óptimas.

El análisis de estequiometría fue hecho con la ecuación (1). Cuando se adicionó $0.4 \mathrm{~g} / \mathrm{L}$ de $\mathrm{MgCl}_{2}$ el reactivo en exceso fue el $\mathrm{NaOH}$, lo que indicó que quedó sin reaccionar 0.11 g, $0.15 \mathrm{~g}$ y $0.09 \mathrm{~g}$ de $\mathrm{NaOH}$ en las soluciones de colorantes: negro elegante, azul profundo y café tabaco, respectivamente. Mientras que cuando se añadió $1.8 \mathrm{~g} / \mathrm{L}$ de $\mathrm{MgCl}_{2}$, el reactivo en exceso fue el $\mathrm{MgCl}_{2}$, y quedó sin reaccionar $1.32 \mathrm{~g}, 1.22$ g y $1.19 \mathrm{~g}$ de $\mathrm{MgCl}_{2}$ en las soluciones de colorantes: negro elegante, azul profundo y café tabaco, respectivamente. Lo anterior indica que se puede adicionar una menor cantidad de $\mathrm{MgCl}_{2}$, dando lugar a un valor de $\mathrm{pH}$ diferente a los obtenidos.

$$
2 \mathrm{NaOH}+\mathrm{MgCl}_{2} \rightarrow 2 \mathrm{NaCl}+\mathrm{Mg}(\mathrm{OH})_{2}
$$

Para el análisis espectral se realizó un scan en el espectrofotómetro marca varian, modelo Cary I con la finalidad de observar en qué longitud de onda se obtenía el pico del colorante (gráfica 1). La longitud de onda máxima del colorante color azul profundo fue de $512 \mathrm{~nm}$ en este estudio, la reportada por Bao-Yu et al. (2007) para el azul (K-GL) fue de $598 \mathrm{~nm}$ y la obtenida por Hai Tan et al. (2000) y Bouyakoub et al. (2011) para el azul brillante EBRA fue de $600 \mathrm{~nm}$. En el colorante negro elegante estudiado la longitud de onda máxima fue de $494 \mathrm{~nm}$. Khayet et al. (2011) obtuvieron que la longitud de onda máxima del negro ácido 210 fue de 460 $\mathrm{nm}$. En el colorante café tabaco la longitud de onda máxima fue de $409 \mathrm{~nm}$; esta longitud de onda no coincide con la que corresponde al Bismarck brown Y (457 nm). Los colorantes estudiados estuvieron en el rango de la longitud de onda máxima reportada por los autores que se mencionaron.

La reducción de la presencia de colorante en el análisis espectral se puede observar en la gráfica 1 para los tres colorantes con los que se experimentó. Se puede apreciar que cuando se adicionó 0.4 y $1.8 \mathrm{mg} / \mathrm{L}$ de $\mathrm{MgCl}_{2}$ el pico en las gráficas que identifica la presencia del colorante desapareció prácticamente.

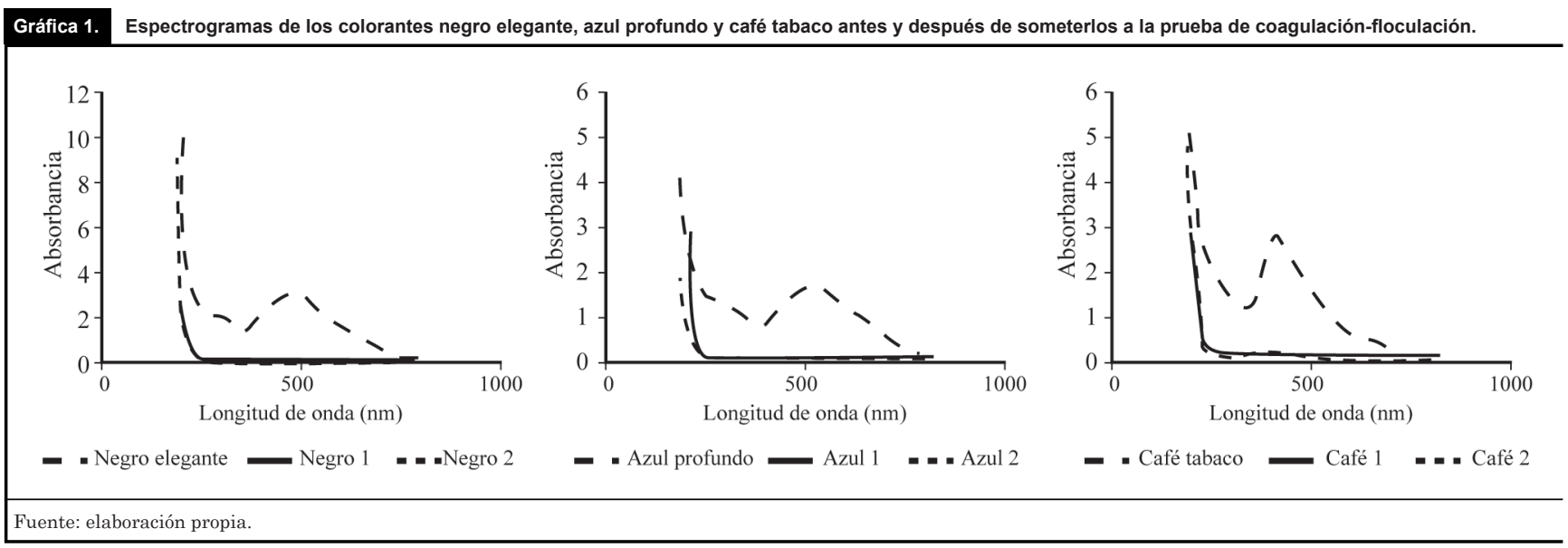


Los resultados obtenidos se muestran el cuadro 2, donde se expone que hay un mayor porcentaje de reducción cuando se adicionó más cantidad de $\mathrm{MgCl}_{2}$. La diferencia entre los porcentajes de reducción de color y DQO obtenidos cuando se adicionó 0.4 y $1.8 \mathrm{~g} / \mathrm{L} \mathrm{MgCl}_{2}$ fue inferior de $6 \%$ para el

Cuadro 2. Concentraciones y porcentajes obtenidos antes y después de realizar las pruebas de coagulaciónfloculación a los colorantes comerciales.

\begin{tabular}{|c|c|c|c|c|c|c|c|}
\hline \multirow[b]{2}{*}{ Muestra } & \multirow[b]{2}{*}{$\begin{array}{c}\mathrm{pH} \\
\text { inicial }\end{array}$} & \multirow[b]{2}{*}{$\begin{array}{c}\mathrm{pH} \\
\text { final }\end{array}$} & \multirow[b]{2}{*}{$\begin{array}{l}\text { Color } \\
(\mathrm{Pt} / \mathrm{Co})\end{array}$} & \multirow[b]{2}{*}{$\begin{array}{l}\text { DQO } \\
(\mathrm{mg} / \mathrm{L})\end{array}$} & \multirow[b]{2}{*}{$\begin{array}{c}\mathrm{SST} \\
(\mathrm{mg} / \mathrm{L})\end{array}$} & \multicolumn{2}{|c|}{ Porcentajes de reducción } \\
\hline & & & & & & Color & DQO \\
\hline Negro elegante & 7.16 & NA & 5380 & 142 & 0 & NA & NA \\
\hline Negro 1 & 11.70 & 10.72 & 362 & 38 & 11.43 & 93.27 & 73.24 \\
\hline Negro 2 & 11.52 & 4.46 & 185 & 27 & 18.57 & 96.56 & 80.99 \\
\hline Azul profundo & 7.03 & NA & 2580 & 80 & 1.43 & NA & NA \\
\hline Azul 1 & 11.83 & 10.91 & 197 & 37 & 64.29 & 92.36 & 53.75 \\
\hline Azul 2 & 11.70 & 4.59 & 73 & 27 & 8.57 & 97.17 & 66.25 \\
\hline Café tabaco & 6.97 & NA & 4640 & 114 & 4.29 & NA & NA \\
\hline Café1 & 11.73 & 10.79 & 464 & 35 & 32.86 & 90.00 & 69.30 \\
\hline Café 2 & 11.73 & 4.68 & 263 & 32 & 18.57 & 94.33 & 71.93 \\
\hline
\end{tabular}

Notas: DQO (demanda química de oxígeno), SST (sólidos suspendidos totales), NA = no aplica. Fuente: elaboración propia.

Figura 1. Muestras de las soluciones de colorantes después de realizar la prueba de coagulación-floculación, dejar sedimentar por 15 y 30 min. y filtrar por carbón activado comercial.

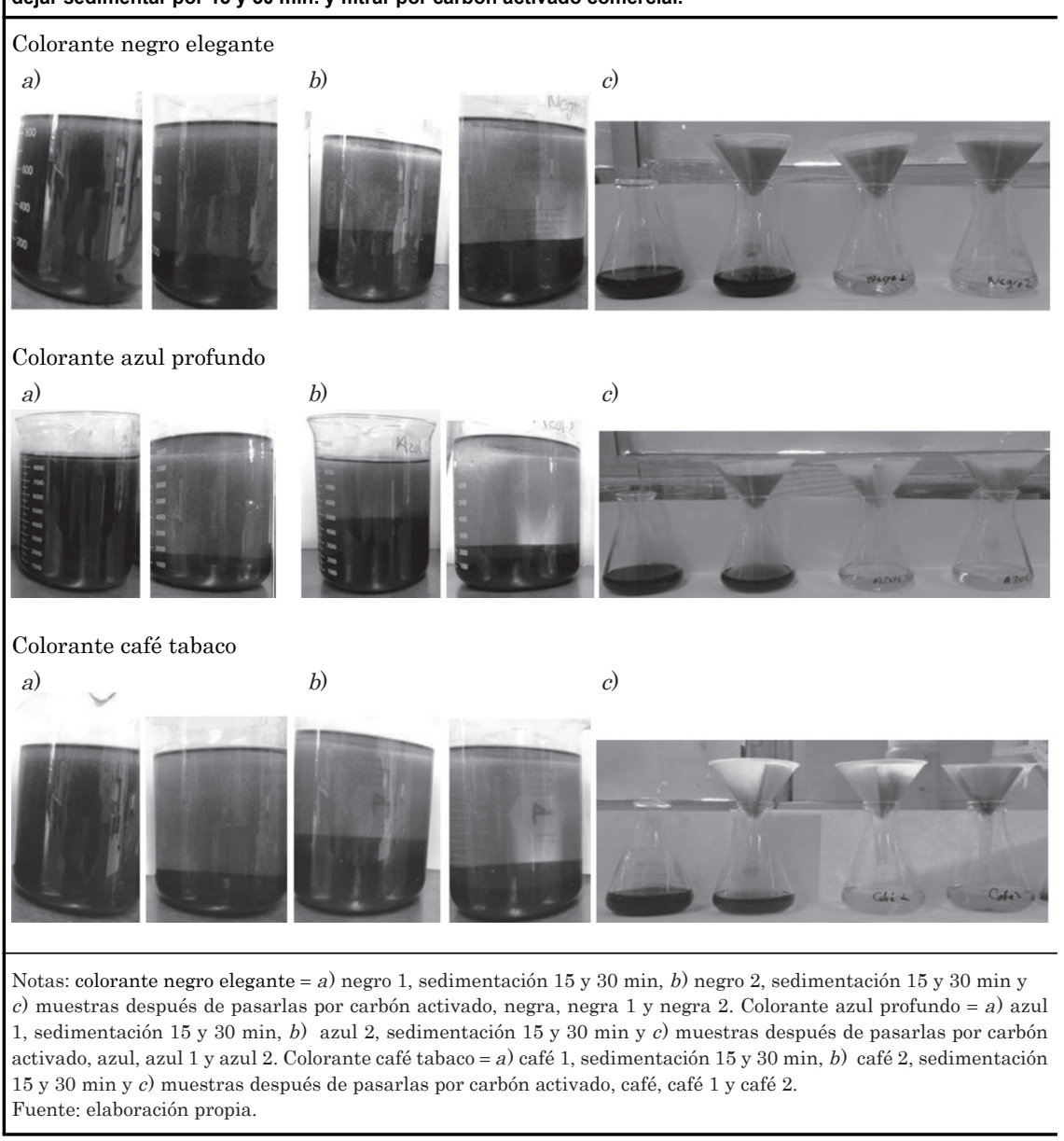

color y menor de 13 \% para la DQO. Los sólidos suspendidos totales aumentaron con la adición de $\mathrm{MgCl}_{2}$.

Las soluciones de colorantes después de ser sometidas al proceso de coagulación-floculación mostraron porcentajes de reducción de color $\geq 90 \%$; sin embargo, el color permaneció.

Con la adición de carbón activado comercial se eliminó el color residual como se aprecia en la figura 1. Esta prueba se hizo adicional con la finalidad de distinguir la reducción de color visualmente. Bao-Yu et al. (2007) centrifugaban el sobrenadante de la muestra, método que no se consideró viable para aplicarlo a escala real.

El carbón activado comercial es costoso por lo que se debe buscar una alternativa económica como la utilizada por Elizalde-González et al. (2006), quienes caracterizaron un adsorbente preparado de desechos de maíz con el fin de utilizarlo en la adsorción de tres tipos de colorantes textiles: ácido, básico y reactivo, lo que representa una opción de sustituir el carbón activado comercial, de ser viable el adsorbente de maíz, para reducir el color de un colorante específico.

\section{Conclusiones}

a) Los resultados de las pruebas de coagulación-floculación indican que el $\mathrm{MgCl}_{2}$ puede reducir el color producido por los colorantes comerciales a través de mecanismos adsortivos con el $\mathrm{Mg}(\mathrm{OH})_{2}$ formado en el proceso de coagulación-floculación.

b) El color no fue reducido totalmente por lo que se requiere un postratamiento, como la adsorción con carbón activado.

c) Si se adiciona una dosis intermedia entre 0.4 y $1.8 \mathrm{~g} / \mathrm{L}$ de $\mathrm{MgCl}_{2}$ se puede llegar a un $\mathrm{pH}$ final de 7.0 y así otro mecanismo de reducción de color sería a través de cargas de neutralización. 


\section{Sugerencias y prospectiva}

Las pruebas realizadas de coagulación-floculación con las soluciones de los colorantes comerciales utilizando $\mathrm{MgCl}_{2}$ permitieron visualizar que, aun utilizando una concentración cuatro veces mayor a la calculada por estequiometría, el color en la solución permanece.

La ventaja de utilizar el $\mathrm{MgCl}_{2}$ es que posteriormente el magnesio puede ser recobrado del precipitado y reciclado en el proceso de coagulación-floculación. La recuperación del magnesio permite apoyar el uso de este proceso en la reducción de color en soluciones con colorantes; no obstante, tiene las desventajas de requerir un control de $\mathrm{pH}$ y la disposición del lodo generado.

También ha de considerarse que la manipulación continua del $\mathrm{MgCl}_{2}$ sin precauciones afecta la salud: en los ojos y la piel puede causar irritación. Si se inhala puede irritar nariz, garganta y tracto respiratorio. La ingestión ocasiona problemas digestivos, náuseas o vómitos. Las complicaciones mencionadas se encuentran en la hoja técnica del producto.

Por otro lado, la prueba adicional de adsorción con carbón activado corroboró que es un método efectivo para tratar aguas residuales con diferentes clases de colorantes; sin embargo, la desventaja es su costo. De recurrir a carbón activado generado a bajo costo, el método de adsorción con carbón activado es viable.

Manejar la prueba de coagulación-floculación con el $\mathrm{MgCl}_{2}$ y posteriormente el carbón activado permitiría prolongar la vida útil del carbón activado.

El uso del $\mathrm{MgCl}_{2}$ y del carbón activado genera residuos de los cuales se tiene que disponer posteriormente. El segundo puede ser regenerado, pero ha de tenerse en consideración que todo producto tiene una vida útil.

Al utilizar las pruebas de coagulación-floculación o adsorción con carbón activado de bajo costo en el proceso de tratamiento de aguas residuales en la industria textil a pequeña escala, se reduciría la contaminación por colorantes en los puntos de descarga. Por este motivo, una sugerencia sería llevar a cabo investigaciones con carbón activado de bajo costo para la reducción de color en soluciones con colorantes.

El lodo generado en el proceso de coagulación-floculación y el carbón activado saturado se pueden calcinar para reducir su volumen y después confinar a un espacio restringido.

Referencias

Bao-Yu, G., Qin-Yan, Y., Yan, W. y Wei-Zhi, Z.

(2007). Color removal from dye-containing wastewater by magnesium chloride. Journal of Environmental Management, 82(2), 167-172.

Bouyakoub, A. Z, Lartiges, B., Ouhib, R., Kacha, S., El Samrani, A. G., Ghanbaja, J., Barres, O. (2011). $\mathrm{MnCl}_{2}$ and $\mathrm{MgCl}_{2}$ for the removal of reactive dye Levafix Brilliant Blue EBRA from synthetic textile wastewaters: An adsorption/ aggregation mechanism. Journal of Hazardous Materials, 187(1-3), 264-273.

DOF (Diario Oficial de la Federación) (2011).

NMX-AA-008-SCFI-2011. Análisis de aguaDeterminación del pH-Método de prueba. DOF (Diario Oficial de la Federación) (2013).

NMX-AA-030/2-SCFI-2001. Análisis de agua -Determinación de la Demanda Química de Oxígeno en aguas naturales, residuales y residuales tratadas-Método de prueba.

DOF (Diario Oficial de la Federación) (2016).

NMX-AA-034-SCFI-2015. Análisis de agua -Determinación de sólidos y sales disueltas en aguas naturales, residuales y residuales tratadas-Método de prueba.

Elizalde-González, M. P., Geyer, W., GuevaraVilla, M. R. G., Mattusch, J., Peláez-Cid, A. A. y Wennrich, R. (2006). Characterization of an adsorbent prepared from maize waste and adsorption of three classes of textile dyes. Colloids and Surfaces A Pbysicochem ical and Engineering Aspects, 278, 89-97.

Faria, P. C. C., Órfão, J. J. M. y Pereira, M. F. R. (2009). Activated carbon and ceria catalysts applied to the catalytic ozonationof dyes and textile effluents. Applied Catalysis B: Environmental, 88, 341-350.

Hach. (2014). Water Analysis Handbook. usA: Hach.

Hai Tan, B., Tow Teng, T. y Mohd Omar, A. K. (2000). Removal of dyes and industrial dye wastes by magnesium chloride. Water Resources, 34(2), 597-601.

Gomathi, D. L., Anantha Raju, K. S., Girish Kumar, S. y Eraiah Rajashekhar, K. (2011). Photo- degradation of di azo dye Bismarck brown by advanced photo-Fenton process: Influence of inorganic anions and evaluation of recycling efficiency of iron powder. Journal of the Taiwan Institute of Chemical Engineers, 42(2), 341-349.

Khayet, M., Zahrim, A. Y. y Hilal, N. (2011) Modelling and optimization of coagulation of highly concentrated industrial grade leather dye by response surface methodology. Chemical Engineering Journal, 167(1), 77-83.

Macy, R. (2005). Química orgánica simplificada. Barcelona: Reverté.

Mittal, J., Thakur, V. y Mittal, A. (2013). Batch removal of hazardous azo dye Bismark brown $\mathrm{R}$ using waste material hen feather. Ecological Engineering, 60, 249-253

Somasiri, W., Xiu-Fen, L., Wen-Quan, R. y Chen, J. (2008). Evaluation of the efficacy of upflow anaerobic sludge blanket reactor in removal of colour and reduction of $\mathrm{COD}$ in real textile wastewater. Bioresource Technology, 99(9), 3692-3699. 
Características de algunos colorantes similares a los utilizados en este estudio.

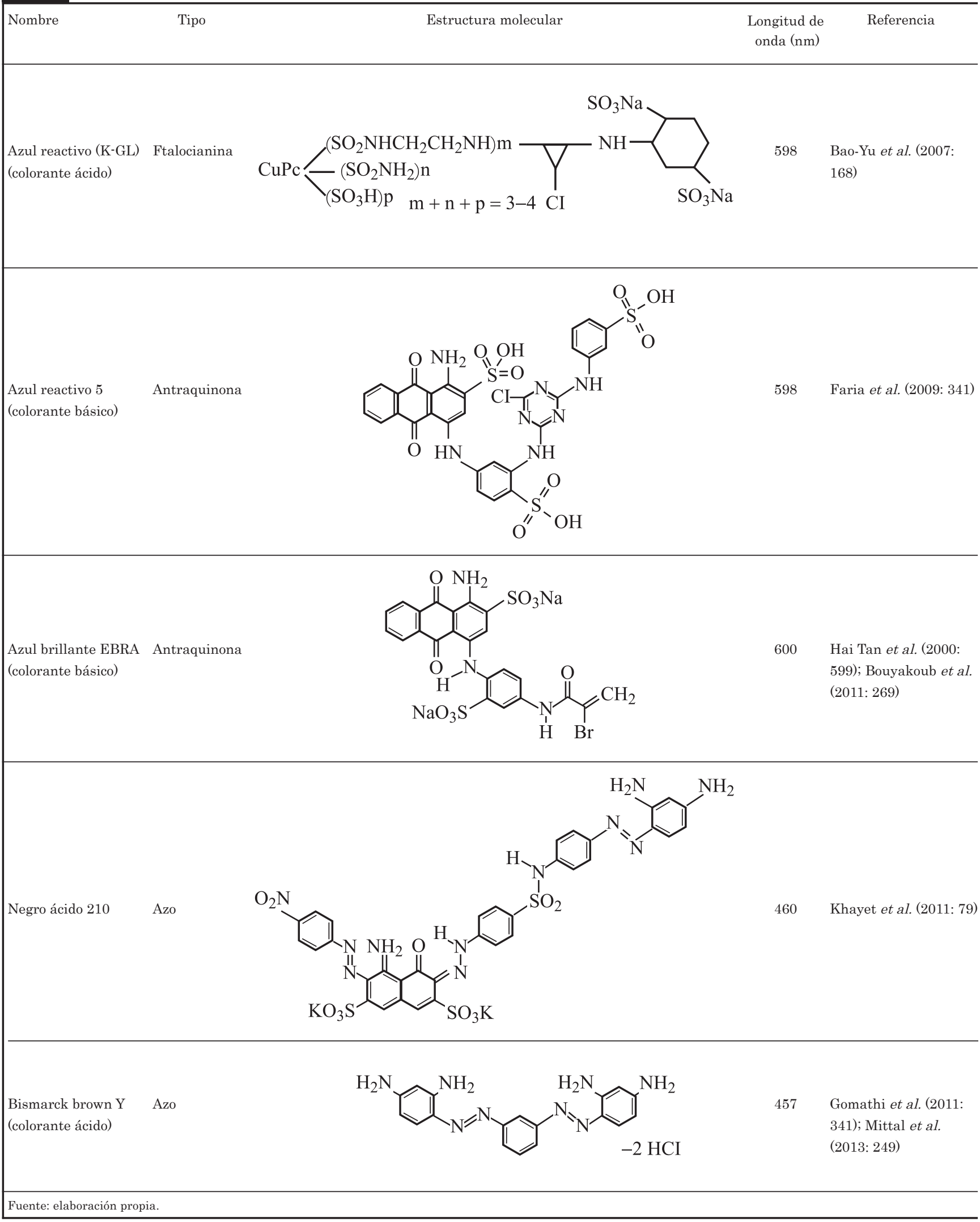

